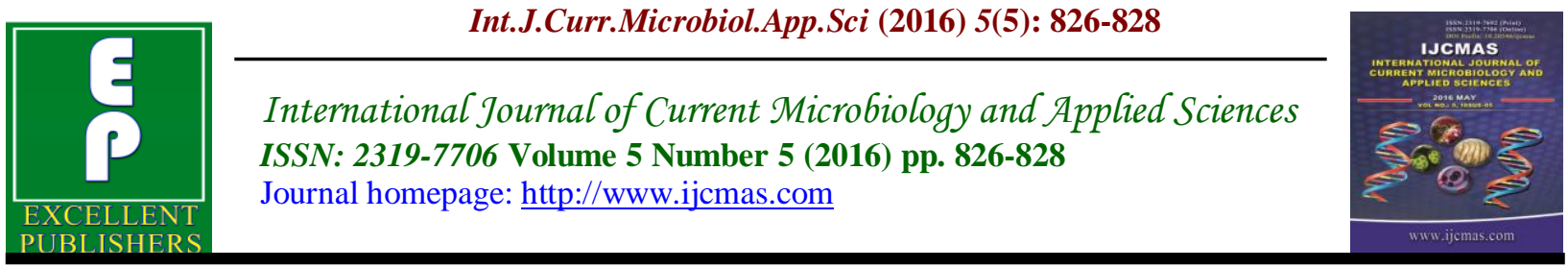

Case Study

http://dx.doi.org/10.20546/ijcmas.2016.505.084

\title{
Tubo-Ovarian Mass-A Rare case of Mycobacterium tuberculosis Infection
}

\author{
S. Latha Roy*, D. R. Gayathri Devi and B. K. Sujini \\ Department of Microbiology, M S Ramaiah Medical College, Bangalore, Karnataka, India \\ *Corresponding author
}

Keywords

Genital

Tuberculosis,

Tuberculosis, Ovary, infertility.

\begin{tabular}{l}
\hline Article Info \\
\hline Accepted: \\
20 April 2016 \\
Available Online: \\
10 May 2016
\end{tabular}

A B S T R A C T

A nulliparous married female, aged 21 years, visited gynecologic OPD of the MS RMC with the complaints of pain abdomen and bleeding P/V since 1 month. PA examination revealed a soft to firm pelvic mass on left side. On vaginal examination, a large mass was palpated on left side which is firm in consistency. Severe anemia $(\mathrm{Hb}-6.5 \mathrm{~g} / \mathrm{dL})$ and high ESR.USG of whole abdomen reveals a heterogeneous lesion in left adnexa mass measuring $102 \times 57 \times 54 \mathrm{~mm}$. Patient underwent USG guided aspiration of ovarian cyst. ZN Stain showed few acid fast bacilli. TB of the female genital tract is nearly always secondary to a focus elsewhere in the body. The clinical diagnosis of genital TB requires a high index of suspicion. The most common initial symptom of genital TB is infertility followed by menstrual disorders.

\section{Introduction}

Genital tuberculosis (TB) in females is not common, particularly in communities where pulmonary or other forms of extragenital TB are endemic. TB can involve any organ system in the body and can present without any clinical manifestation. Genital TB may be asymptomatic and diagnosis requires a high index of suspicion. ${ }^{2}$

Female genital TB is a disease of young women in the age group of 20-40 years with $80-90 \%$ of cases diagnosed during the workup for infertility. ${ }^{4}$

Genital TB develops from dissemination of Mycobacterium from other site of the body usually pulmonary and sometimes renal, gastrointestinal, bone, joint, or it may be a part of a generalized miliary disease process.
The criteria necessary for a diagnosis of primary genital TB incudes are: (1) The genital TB lesions should be the first TB infection in the body, and (2) regional lymph nodes should demonstrate the same stage of TB development as do the genital organs.

\section{Case Report}

A nulliparous married female, aged 21 years, visited gynecologic out-patient department of the MS Ramaiah hospital with the complaints of mass per abdomen since 1 month. She also complained of bleeding P/V since 1 month associated with lower abdominal pain. She had received the Bacille Calmette-Guerin vaccination at birth and there was no history of contact with any case of TB. No long-term history of medication was present. 


\section{Investigation}

Per abdominal examination revealed a soft to firm pelvic mass (size 2 weeks) on left side.

On vaginal examination, a large mass was palpated on left side which is firm in consistency. The mass was tender on palpation. Blood tests showed a severe anemia with a hemoglobin $6.5 \mathrm{~g} / \mathrm{dL}$, total leukocyte count- $8920 / \mathrm{mm} 3$, platelet count3.88 lakhs $/ \mathrm{mm}^{3}$ and erythrocyte sedimentation rate- $43 \mathrm{~mm} / \mathrm{hr}$. Bleeding and clotting time were normal. VDRL and HIV were non-reactive.

Ultrasonography of whole abdomen reveals a heterogeneous lesion in left adnexa mass measuring $102 \times 57 \times 54 \mathrm{~mm}$. The provisional diagnosis of Tuboovarian mass was made.

Patient underwent USG guided aspiration of ovarian cyst. The pus was thick, purulent non foul smelling, not blood stained. Gram's stain showed numerous inflammatory cells with no organism. ZN Stain showed few acid fast bacilli. Patient referred to RNTCP and started on ATT. Routine aerobic and anaerobic culture remains sterile. Rough Buff colored growth was seen on LJ media after 5 weeks. MPT 64 test confirmed positive for Mycobacterium complex. The sensitivity was done at Elbit diagnostics and is sensitive to all antibiotics.

\section{Discussion}

TB of the female genital tract is nearly always secondary to a focus elsewhere in the body.3The TB bacilli reach the genital tract by three principal routes. Haematogenous spread represents about $90 \%$ of cases, with the primary focus being the lungs, lymph nodes or skeletal system. Descending direct spread occurs, with infection reaching the genital organs via the lymphatic system or directly from the gastrointestinal tract, mesenteric nodes or the peritoneum.3,5 Approximately $50 \%$ of patients might have had pulmonary TB, TB pleurisy, peritonitis, erythema nodosum, or renal, osseous TB. A history of primary infertility in a woman in whom examination reveals no apparent cause and who gives a family history or personal history of TB of any organ should arouse suspicion of genital TB.

The most common initial symptom of genital TB is infertility. Lower abdominal pain and menstrual disorders are the other most common symptoms after infertility. 10$40 \%$ patient of genital TB have abnormal uterine bleeding. Menorrhagia, menometrorrhagia, intermenstrual bleeding, oligomenorrhea, and postmenopausal bleeding may be present. 1

Infertility for which no obvious cause can be found, chronic pelvic inflammatory disease refractory to standard antibiotic therapy, or adnexal disease with ascites in virgin females should alert the clinician to look for TB of the genital tract. 1

\section{Conclusion}

Genital TB in females is one of the most important causes of infertility so the possibility of TB infection of the genital tract should always be considered especially in a patient from an area where $\mathrm{TB}$ is endemic.

\section{References}

1. Raviglione MC, O'Brien JR. Harrison's Principles of Internal Medicine. 18th ed., Ch. 165. New York: McGraw Hill; 2012. p. 1340-60.

2. Cow TWP, Lim KB, Valliparim S. The masquerades of female pelvic tuberculosis: case reports and review of literature on clinical presentation and 
diagnosis. J Obstet Gynaecol Res 2002; 28:203-10.

3. Qureshi RN, Sammad S, Hamd R, Lakha SF. Female genital tuberculosis revisited. J Pak Med Assoc2001;51:1618.

4. Schaefer G. Female genital tuberculosis. Clin Obstet Gynecol 1976; 19:223 39 .
5. David K Gatongi, Godfrey Gitau, Vanessa Kay Female genital tuberculosis, The Obstetrician \& Gynaecologist 2005;7:75-79.

\section{How to cite this article:}

Latha Roy, S., D. R. Gayathri Devi and Sujini, B. K. 2016. Tubo-Ovarian Mass-A Rare case of Mycobacterium tuberculosis Infection. Int.J.Curr.Microbiol.App.Sci.5(5): 826-828. doi: http://dx.doi.org/10.20546/ijcmas.2016.505.084 